\title{
Novel BCL6 Inhibitor (FX1): Advances in Diffuse Large B-Cell Lymphomas (DLBCLs) Treatment
}

\author{
Md Kamrul Hasan \\ Moores Cancer Center, University of California San Diego, La Jolla, USA
}

Email address:

k0hasan@ucsd.edu

\section{To cite this article:}

Md Kamrul Hasan. Novel BCL6 Inhibitor (FX1): Advances in Diffuse Large B-Cell Lymphomas (DLBCLs) Treatment. Biomedical Sciences. Vol. 3, No. 2, 2017, pp. 55-57. doi: 10.11648/j.bs.20170302.12

Received: November 25, 2016; Accepted: February 13, 2017; Published: April 26, 2017

\begin{abstract}
Diffuse large B-cell lymphomas (DLBCLs) are the most common and aggressive type of all the lymphomas. B cell lymphoma 6 (BCLO) oncogene is highly expressed in DLBCLs and one of the major reasons of treatment failure. Recent findings suggest that specific BCL6 inhibitor (FX1) disrupted formation of the BCL6 repression complex, reactivated BCL6 target genes, that in-turn suppressed growth of DLBCLs cells, primary human DLBCLs specimens, as well as induced regression of established tumors in mice derived from DLBCLs cells.
\end{abstract}

Keywords: Diffuse Large B-Cell Lymphomas (DLBCLs), BCL6, FX1

\section{Introduction}

Diffuse large B-cell lymphomas (DLBCLs) are an aggressive type of non-Hodgkin lymphoma that develops from the B-cells in the lymphatic system and accounts for $30 \%-40 \%$ of all cases. The disease is heterogeneous clinically, morphologically, molecularly; and characteristically exhibit poor prognosis [1]. There are two major biologically distinct molecular subtypes of DLBCLs: (i) germinal center B-cell (GCB-DLBCL) and (ii) activated B-cell (ABC-DLBCL). Although, the R-CHOP (cyclophosphamide, doxorubicin, vincristine, and prednisone; plus the monoclonal antibody rituximab) regimen remains the standard first-line treatment for patients with DLBCLs; however over $40 \%$ of patients relapse and die of their disease [2-5]. Patients with ABC-DLBCL observes more unfavorable clinical outcome as compared with GCB-DLBCL [6, 7].

BCL6 induces proliferation and survival of GC-B cells through repressing various checkpoint pathways, by recruiting the SMRT (silencing mediator for retinoid or thyroid-hormone receptors), NCOR (Nuclear Receptor Corepressor), and BCOR (BCL-6 corepressor) corepressors to its $N$-terminal BTB domain $[8,9]$. The binding site for these corepressors consists of an extended groove formed through obligate homodimerization of BTB domains of BCL6, and mice expressing BCL6 with mutation in the corepressor-binding groove fail to form GCs [10-12]. Moreover, translocations and amplifications of the B cell lymphoma 6 (BCL6) locus causes high expression BCL6 protein in ABC-DLBCL [13-15].

\section{Results}

Recently, Cardenas M. G. et al. identified FX1 as a specific BCL6 BTB inhibitor by using an in silico drug design functional-group mapping approach called SILCS, that has greater potency than endogenous corepressors and binds an essential region of the BCL6 lateral groove [16]. FX1 activity was then screened using a BCL6 $6^{\mathrm{BTB}}$-GAL4 DNA binding domain luciferase reporter assay as well as Monte CarloSILCS (MC-SILCS)-ligand grid free energy (LGFE) analysis, and found that FX1 as the most active and selective BCL6 BTB inhibitor. Microscale thermophoresis (MST) analysis revealed that FX1 bound to BCL6 BTB domain with over 4-fold higher affinity than its natural corepressor SMART, and over 18-fold greater affinity than previously reported BCL6 inhibitor 79-6 [17].

In reporter assays, FX1 exhibited 10-fold greater inhibitory activity against the BCL6 BTB domain compared to related BTB domain containing transcription factors, and biochemical enzymatic activity analysis showed that FX1 at concentration of $10 \mu \mathrm{M}$ inhibits $80 \%$ of BCL6 activity without significantly inhibiting other 50 kinases, confirming that FX1 binding is 
selective to BCL6. By inhibiting BCL6 to associate with its coreperessors, FX1 can induce BCL6 regulated target genes including CASP8 (Caspase-8), CD69 (Cluster of Differentiation 69), CXCR4 (C-X-C chemokine receptor type 4), CDKN1A (Cyclin-Dependent Kinase Inhibitor 1A), and DUSP5 (Dual Specificity Phosphatase 5).

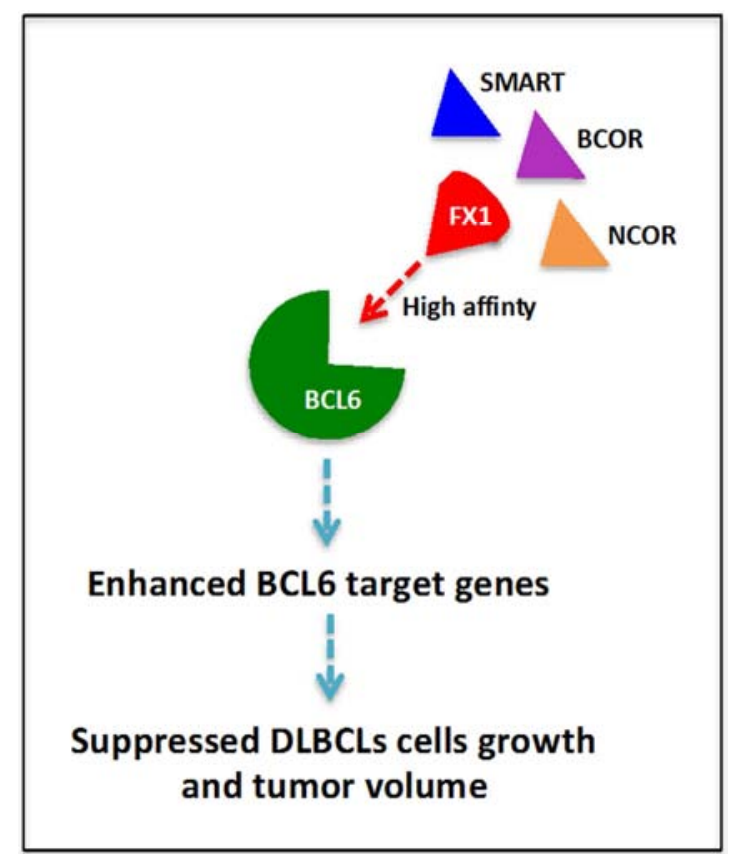

Figure 1. Schematic diagram of model for how FX1 can competitively bind to BCL6 and inhibit its corepressors such as SMART, BCOR, NCOR; that reactivate BCL6 target genes, which in-turn suppressed DLBCLs cells growth and tumor volume. Courtesy of Cardenas M. G. et al. (modified by M. K. Hasan).

In-vitro and in-vivo analysis revealed that FX1 showed a selective growth inhibition effect on BCL6-dependent GCB-DLBCL cell lines with average $\mathrm{GI}_{50}$ values of about 36 $\mu \mathrm{M}$, and FX1 caused profound and significant suppression of DLBCL xenografts in mice without exhibiting any toxic effects, and indeed not only prevented growth of the xenografts but in addition caused these tumors to shrink from their initial volume.

Although, ABC-DLBCL is relatively more resistance to $\mathrm{CHOP}$ plus rituximab (R-CHOP) therapy compared with GCB-DLBCL $^{7}$; FX1 showed potential sensitivity to ABC-DLBCL cell lines with an average $\mathrm{IC}_{50}$ of $41 \mu \mathrm{M}$ and suppressed xenograft tumors derived from ABC-DLBCL cell line. Moreover, FX1 significantly decreased the survival of primary human DLBCLs cells including GCB-DLBCL and $\mathrm{ABC}$-DLBCL origin. In addition, combined treatment of FX1 with the chemotherapeutic drug doxorubicin showed that FX1 enhances response to doxorubicin in GCB-DLBCL as well as the more chemotherapy-resistant ABC-DLBCL.

\section{Conclusion}

Overall results suggesting that FX1 might have potential applications for the treatment of patients with DLBCLs including GCB-DLBCL and ABC-DLBCL.

\section{Abbreviations}

DLBCLs: Diffuse large B-cell lymphomas; BCL6: B cell lymphoma 6; GCB-DLBCL: Germinal center B-cell DLBCL; ABC-DLBCL: Activated B-cell DLBCL; R-CHOP: cyclophosphamide, doxorubicin, vincristine, and prednisone; plus the monoclonal antibody rituximab; SMRT: Silencing mediator for retinoid or thyroid-hormone receptors; NCOR: Nuclear receptor corepressor; BCOR: BCL-6 corepressor; MC-SILCS: Monte carlo-SILCS; LGFE: Ligand grid free energy; CASP8: Caspase-8; CD69: Cluster of differentiation 69; CXCR4: C-X-C chemokine receptor type 4, CDKN1A: Cyclin-dependent kinase inhibitor 1A; DUSP5: Dual specificity phosphatase 5 .

\section{References}

[1] Sehn LH, Gascoyne RD. Diffuse large B-cell lymphoma: optimizing outcome in the context of clinical and biologic heterogeneity. Blood 2015 Jan 1; 125(1): 22-32.

[2] Coiffier B, Lepage E, Briere J, Herbrecht R, Tilly H, Bouabdallah R, et al. CHOP chemotherapy plus rituximab compared with CHOP alone in elderly patients with diffuse large-B-cell lymphoma. The New England journal of medicine 2002 Jan 24; 346(4): 235-242.

[3] Habermann TM, Weller EA, Morrison VA, Gascoyne RD, Cassileth PA, Cohn JB, et al. Rituximab-CHOP versus CHOP alone or with maintenance rituximab in older patients with diffuse large B-cell lymphoma. Journal of clinical oncology: official journal of the American Society of Clinical Oncology 2006 Jul 1; 24(19): 3121-3127.

[4] Pfreundschuh M, Kuhnt E, Trumper L, Osterborg A, Trneny M, Shepherd L, et al. CHOP-like chemotherapy with or without rituximab in young patients with good-prognosis diffuse large-B-cell lymphoma: 6-year results of an open-label randomised study of the MabThera International Trial (MInT) Group. The Lancet Oncology 2011 Oct; 12(11): 1013-1022.

[5] Sehn LH, Donaldson J, Chhanabhai M, Fitzgerald C, Gill K, Klasa R, et al. Introduction of combined CHOP plus rituximab therapy dramatically improved outcome of diffuse large B-cell lymphoma in British Columbia. Journal of clinical oncology: official journal of the American Society of Clinical Oncology 2005 Aug 1; 23(22): 5027-5033.

[6] Alizadeh AA, Eisen MB, Davis RE, Ma C, Lossos IS, Rosenwald A, et al. Distinct types of diffuse large B-cell lymphoma identified by gene expression profiling. Nature 2000 Feb 3; 403(6769): 503-511.

[7] Lenz G, Staudt LM. Aggressive lymphomas. The New England journal of medicine 2010 Apr 15; 362(15): 1417-1429.

[8] Huynh KD, Bardwell VJ. The BCL-6 POZ domain and other POZ domains interact with the co-repressors N-CoR and SMRT. Oncogene 1998 Nov 12; 17(19): 2473-2484.

[9] Huynh KD, Fischle W, Verdin E, Bardwell VJ. BCoR, a novel corepressor involved in BCL-6 repression. Genes \& development $2000 \mathrm{Jul}$ 15; 14(14): 1810-1823. 
[10] Ahmad KF, Melnick A, Lax S, Bouchard D, Liu J, Kiang CL, et al. Mechanism of SMRT corepressor recruitment by the BCL6 BTB domain. Molecular cell 2003 Dec; 12(6): 1551-1564.

[11] Ghetu AF, Corcoran CM, Cerchietti L, Bardwell VJ, Melnick A, Prive GG. Structure of a BCOR corepressor peptide in complex with the BCL6 BTB domain dimer. Molecular cell 2008 Feb 15; 29(3): 384-391.

[12] Huang C, Hatzi K, Melnick A. Lineage-specific functions of Bcl-6 in immunity and inflammation are mediated by distinct biochemical mechanisms. Nature immunology 2013 Apr; 14(4): 380-388.

[13] Iqbal J, Greiner TC, Patel K, Dave BJ, Smith L, Ji J, et al. Distinctive patterns of BCL6 molecular alterations and their functional consequences in different subgroups of diffuse large B-cell lymphoma. Leukemia 2007 Nov; 21(11): 2332-2343.

[14] Bea S, Zettl A, Wright G, Salaverria I, Jehn P, Moreno V, et al.
Diffuse large B-cell lymphoma subgroups have distinct genetic profiles that influence tumor biology and improve gene-expression-based survival prediction. Blood 2005 Nov 1; 106(9): 3183-3190.

[15] Green MR, Vicente-Duenas C, Romero-Camarero I, Long Liu C, Dai B, Gonzalez-Herrero I, et al. Transient expression of Bcl6 is sufficient for oncogenic function and induction of mature B-cell lymphoma. Nature communications 2014; 5: 3904.

[16] Cardenas MG, Yu W, Beguelin W, Teater MR, Geng H, Goldstein RL, et al. Rationally designed BCL6 inhibitors target activated B cell diffuse large B cell lymphoma. The Journal of clinical investigation 2016 Sep 1; 126(9): 3351-3362.

[17] Cerchietti LC, Ghetu AF, Zhu X, Da Silva GF, Zhong S, Matthews M, et al. A small-molecule inhibitor of BCL6 kills DLBCL cells in vitro and in vivo. Cancer cell 2010 Apr 13; 17(4): 400-411. 